\title{
Empirical treatment of influenza-associated pneumonia in primary care: a descriptive study of the antimicrobial susceptibility of lower respiratory tract bacteria (England, Wales and Northern Ireland, January 2007-March 2010)
}

\author{
Ruth M Blackburn, ${ }^{1}$ Katherine L Henderson, ${ }^{1}$ Mark Lillie, ${ }^{1}$ Elizabeth Sheridan, ${ }^{1}$ \\ Robert C George, ${ }^{2}$ Adrian H B Deas, ${ }^{3}$ Alan P Johnson ${ }^{1}$
}

- An additional table is published online only. To view this file please visit the journal online (http://thorax.bmj.com)

${ }^{1}$ Healthcare Associated Infections and Antimicrobial Resistance Department, Health Protection Agency, Centre for Infections, UK

${ }^{2}$ Respiratory \& Systemic Infections Laboratory, Centre for Infections, Health Protection Agency, Centre for Infections, London, UK

${ }^{3}$ Regional Epidemiology Unit, Health Protection Agency (East of England), Institute of Public Health, Cambridge, UK

Correspondence to

Ruth M Blackburn, Healthcare Associated Infections and Antimicrobial Resistance Department, Health Protection Agency, Centre for Infections, 61 Colindale Avenue, London NW9 5E0, UK:

ruth.blackburn@hpa.org.uk

Received 5 January 2010 Revised 30 September 2010 Accepted 6 January 2011 Published Online First 25 February 2011

\section{ABSTRACT}

Objectives To determine the susceptibility of lower respiratory tract (LRT) isolates of Streptococcus pneumoniae, Staphylococcus aureus and Haemophilus influenzae to antimicrobial agents recommended by UK guidelines for treatment of pneumonia associated with influenza-like illness.

Methods Analysis of antimicrobial susceptibility data from sentinel microbiology laboratories in England, Wales and Northern Ireland was carried out. Subjects comprised patients who had an LRT specimen taken in a general practitioner surgery or hospital outpatient setting between January 2007 and March 2010. The main outcome measurements were antimicrobial susceptibility trends of LRT isolates over time, between patient age groups and in different geographical regions. Results Susceptibility to tetracyclines or co-amoxiclav was high. Of the 70288 and 45288 isolates with susceptibility results for tetracyclines or co-amoxiclav, $96 \%$ and $92 \%$, respectively, were susceptible. Overall susceptibility to ciprofloxacin, ampicillin/amoxicillin and macrolides was lower than for tetracyclines or co-amoxiclav and varied markedly by organism. There were few clinically relevant variations in susceptibility to doxycycline or co-amoxiclav over time, geographically or between age groups.

Conclusions The data support the use of doxycycline or co-amoxiclav as appropriate empiric treatment for LRT infection caused by the pathogens investigated, for patients in primary care.

\section{BACKGROUND}

Influenza may be complicated by pneumonia, which has two recognised types: primary viral and secondary bacterial infection. ${ }^{2}$ Examination of pathological specimens from the 1918-1919 influenza pandemic suggested that secondary bacterial infection was the major cause of deaths associated with influenza-like illness (ILI) during that pandemic. ${ }^{3}$ The prognosis of patients presenting with bacterial pneumonia rapidly worsens with delay in treatment, and effective empirical treatment (before or in the absence of a specific microbiological diagnosis) is therefore a critical aspect of successful pandemic influenza planning. ${ }^{4}$

Guidelines for the clinical management of patients with ILI during an influenza pandemic were compiled and published in 2007 by the British
Infection Society, British Thoracic Society and Health Protection Agency (HPA) in collaboration with the Department of Health. ${ }^{1}$ These guidelines regarded the pathogens likely to be associated with secondary bacterial pneumonia as Streptococcus pneumoniae, Staphylococcus aureus and Haemophilus influenzae. The guidelines suggest oral empirical treatment with either a tetracycline (usually doxycycline) or co-amoxiclav for patients in primary care. More recently, Department of Health guidelines introduced specifically for the 2009 H1N1 pandemic have reiterated the use of doxycycline or co-amoxiclav treatment for adult patients with influenza complicated by lower respiratory tract (LRT) infection that are not admitted to hospital. ${ }^{6}$ Co-amoxiclav is recommended for children (except patients with penicillin allergy, who should be given clarithromycin).

This paper seeks to assess microbiological evidence as to whether co-amoxiclav or tetracyclines are appropriate empirical treatment for bacterial pneumonia in patients treated in the primary care setting. The analysis draws on susceptibility data for LRT isolates of $S$ pneumoniae, $S$ aureus and $H$ influenzae as reported to LabBase2, the HPA's national communicable disease database. The database collects isolate data from laboratories across England, Wales and Northern Ireland and was extended to cover LRT isolates in 2006 as part of the pandemic influenza preparedness arrangements. The surveillance aims to support the timely modification of empirical treatment in the event that antimicrobial susceptibility patterns change.

\section{METHODS}

Antimicrobial susceptibility data for LRT isolates of $H$ influenzae, $S$ pneumoniae and $S$ aureus reported between January 2007 and March 2010 were extracted from LabBase2 for laboratories in England, Wales and Northern Ireland. LRT isolates were primarily obtained from sputum, but other sources included alveolar lavage, bronchial/endotracheal aspirate, LRT, lung, pleura or pleural fluid.

The data included all susceptibility test results for tetracycline, co-amoxiclav, ciprofloxacin, ampicillin/amoxicillin and macrolides for all three organisms as reported by hospital laboratories. Data on ciprofloxacin susceptibility in $S$ pneumoniae 
were excluded as ciprofloxacin is known to be ineffective against this organism ${ }^{78}$ and pneumococcal isolates are rarely tested for ciprofloxacin susceptibility. Susceptibility data for macrolides were estimated by pooling results for azithromycin, erythromycin and clarithromycin; where conflicting results were reported for the same isolate for different macrolides, the resistant result was retained in the data set. To classify $S$ aureus as either methicillin resistant (MRSA) or susceptible (MSSA), results for antistaphylococcal $\beta$-lactamase-stable penicillins were pooled. Penicillin was used as a proxy for co-amoxiclav susceptibility in pneumococci due to the small number of test results available for co-amoxiclav. Demographic information was collected, including geographical region, date of birth and isolate source description (ie, general practitioner (GP) surgery, outpatient). The analysis was confined to specimens taken in GP surgeries or from hospital outpatients.

Where multiple successive isolates were reported from the same patient, results for the first isolate only were included in the analysis. Data were analysed using Stata v11 (Stata Corp.). Data for each organism were stratified by the quarter in which the specimen was collected, region, patient age, sex and source. Wilson's method ${ }^{9}$ was used to calculate 95\% CIs (shown graphically as error bars) for the percentage of susceptible isolates.

Two Poisson models with robust standard errors were used in the analysis, with incident rate ratios (IRRs) expressed as the outcome measure. The first of these models explored the major focus of the paper which was evaluating changes in antimicrobial susceptibility over time by organism. The second model examined changes in susceptibility by organism and patient age group. As likelihood ratio testing (for the purposes of model building) is problematic for a model with robust standard errors, the final Poisson models were constructed by identifying key variables and interactions in logistic regression models.

The logistic regression models were constructed using a backwards elimination approach that incorporated categorical variables for organism, age group, quarter (1, January-March; 2, April-June; 3, July-September; 4, October-December), sex, laboratory region and isolate source (GP or outpatient) to measure susceptibility over time in years (coded as a continuous variable). Patients were categorised by age at specimen date as $<45,45-65,65-74$ and $75+$ years, with the group with the largest number of susceptibility results (45-65 years) chosen as the baseline group. The North West was used as the baseline laboratory region because the greatest amount of susceptibility data was reported by this region. Likelihood ratio testing was used to identify variables that could be satisfactorily dropped from each model. Variables were only excluded from the model if the ORs for the remaining variables did not change markedly.
After the variables for inclusion in the models had been determined, possible interactions between either organism and year or organism and patient age group were investigated and, if significant, retained for use in the Poisson model. Organismspecific IRRs for changes over time or by patient age group were obtained by using linear combinations of the appropriate coefficients (using the 'lincom' command).

Data from the National External Quality Assurance Scheme for susceptibility testing (in which all clinical microbiology laboratories in England, Wales and Northern Ireland take part) were reviewed for the three organisms and antibiotics (tetracycline or co-amoxiclav/penicillin).

\section{RESULTS \\ Overview}

A total of 86845 LRT isolates of $H$ influenzae, $S$ pneumoniae and $S$ aureus were identified from specimens reported from GP surgeries or outpatients. Over $96 \%$ of isolates were obtained from sputum, with the remaining isolates obtained from bronchial aspirate $(2.4 \%)$, alveolar lavage $(0.9 \%)$ or endotracheal aspirate, LRT, lung, pleura and pleural fluid specimens $(<1 \%)$. Haemophilus influenzae accounted for the majority (62\%, 53 895) of isolates, with an equal split between $S$ aureus $(19 \%, 16860)$ and $S$ pneumoniae $(19 \%, 16090)$. It should be noted that methicillin susceptibility was not reported for a subset of $962 S$ aureus isolates and therefore the total number of $S$ aureus exceeds the sum of known MRSA (3458, 22\%) and MSSA (12 440, 78\%). A total of 57827 (67\%) specimens were sourced from GP surgeries, with the remaining third of specimens from outpatients. The median age for all patients included in the study was 63.6 years, while the mean age for GP and outpatient specimens was 61.5 and 54.2 years, respectively. A two-sample Wilcoxon rank-sum test confirmed that there was strong evidence of a difference $(p<0.001)$ between the ages of GP patients and hospital outpatients.

The total number of hospital laboratories that reported isolates to the surveillance scheme each year increased during the course of the study from 85 to 99 . The mean annual number of laboratories reporting per country was 87, 4 and 1.3 for England, Wales and Northern Ireland, respectively. The proportion of isolates for which susceptibility results were available varied by antibiotic (range 41-81\%) and was highest for tetracycline (81\%) and co-amoxiclav (64\%). Susceptibility to tetracyclines or co-amoxiclav was high $(96 \%$ and $92 \%$, respectively; table 1). The proportion of $S$ aureus isolates tested for coamoxiclav was $10 \%$ and the estimated $78 \%$ of isolates susceptible to co-amoxiclav should therefore be considered with some caution. As expected all reported MSSA isolates were susceptible. However, there were a small number of MRSA isolates

Table 1 Antimicrobial susceptibility of lower respiratory tract isolates, England, Wales and Northern Ireland (January 2007-March 2010)

\begin{tabular}{|c|c|c|c|c|c|c|}
\hline & \multirow[b]{2}{*}{ Total isolates } & \multicolumn{5}{|c|}{ No. of isolates tested (\% susceptible) } \\
\hline & & Tetracyclines & Co-amoxiclav & Ciprofloxacin & $\begin{array}{l}\text { Ampicillin/ } \\
\text { amoxicillin }\end{array}$ & Macrolides $\dagger$ \\
\hline$H$ influenzae & 53895 & $46342(99)$ & $43685(93)$ & $27175(99)$ & $49834(77)$ & $24583(15)$ \\
\hline$S$ pneumoniae & 16090 & $12876(91)$ & $-*$ & - & 4919 (97) & $14800(87)$ \\
\hline$S$ aureus & 16860 & $11070(94)$ & $1603(78)$ & $8146(61)$ & $1047(23)$ & $15410(68)$ \\
\hline MSSA & 12440 & $8131(95)$ & $1230(100)$ & $5825(83)$ & $836(28)$ & $12230(79)$ \\
\hline MRSA & 3458 & $2665(94)$ & $359(5)$ & $2279(4)$ & $204(2)$ & $2970(24)$ \\
\hline Total & 86845 & $70288(96)$ & $45288(92)$ & $35321(90)$ & $55800(77)$ & 54793 (49) \\
\hline
\end{tabular}

*If benzyl-penicillin is used as a proxy for co-amoxiclav, susceptibility is $95 \%$ (based on 13243 isolates tested).

†Combined susceptibility results for azithromycin, erythromycin and clarithromycin.

MRSA, methicillin-resistant Staphylococcus aureus; MSSA, methicillin-susceptible Staphylococcus aureus. 
that were reported as susceptible to co-amoxiclav, which is likely to be a reporting or testing artefact. In contrast, reports for tetracycline susceptibility in $S$ aureus were robust $(66 \%$ of isolates were tested) and there was little variation in susceptibility between MSSA (95\%) and MRSA (94\%). The overall susceptibility of isolates to ciprofloxacin $(90 \%$-excluding $S$ pneumoniae isolates), ampicillin/amoxicillin (77\%) and macrolides $(49 \%)$ was markedly lower than for tetracyclines or co-amoxiclav.

Co-amoxiclav susceptibility of $S$ pneumoniae was estimated using benzyl-penicillin as a surrogate as pneumococcal isolates are not routinely tested for co-amoxiclav susceptibility and numbers were therefore low. Of the 16090 isolates tested for either penicillin or co-amoxiclav, a total of $1131(7 \%)$ isolates were tested for both antibiotics and, of these, 17 were reported as penicillin resistant but co-amoxiclav susceptible, $1100 \mathrm{had}$ dual susceptibility and the remaining 14 were resistant to both antibiotics. There were no reports of isolates susceptible to penicillin but resistant to co-amoxiclav. It should be noted that $>50 \%$ of the co-amoxiclav susceptibility data available for $S$ pneumoniae were reported by just three laboratories and may therefore not be representative of all sites. However, these
Figure 1 Percentage susceptibility of $H$ influenzae (A), S pneumoniae (B) and $S$ aureus (C) from lower respiratory tract isolates from patients seen by general practitioners and in outpatients between January 2007 and March 2010.
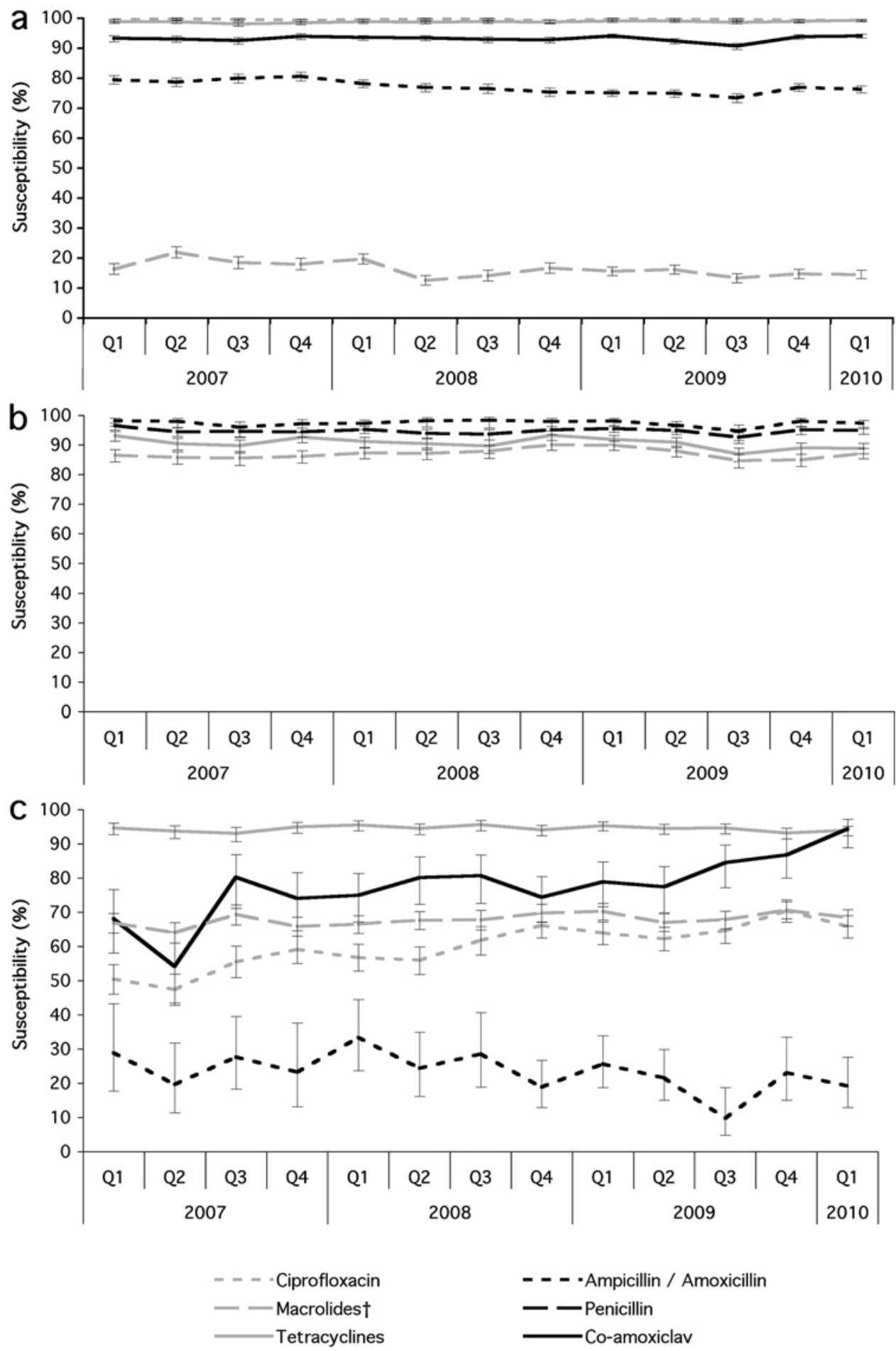

†Combined susceptibility results for azithromycin, erythromycin and clarithromycin 
results suggest that pneumococcal susceptibility to co-amoxiclav may be closer to $99 \%$ than the $95 \%$ estimate based on penicillin susceptibility.

\section{Changes in susceptibility over time}

The percentage susceptibility of each of the three organisms to the antibiotics tested is shown in figure $1 \mathrm{~A}-\mathrm{C}$. In general, changes in susceptibility over time were small. However, $S$ aureus susceptibility to co-amoxiclav in the second quarter of 2007 dipped due to a peak in the relative proportion of MRSA to MSSA (ratio of 1:1.1 for 2007 quarter 2 compared with a mean ratio for the whole time period of 1:3.4) and also reflects the small number of isolates tested for this quarter $(n=85)$.

Table 2 outlines IRRs for the annual rate of susceptibility change for each antibiotic investigated accounting for seasonal variation and other important confounders such as patient age group, sex and source. The results suggest that susceptibility to tetracycline, co-amoxiclav and ciprofloxacin has remained relatively constant for all organisms except $S$ aureus, for which the estimated $9 \%$ annual increase in susceptibility to co-amoxiclav and ciprofloxacin is most likely to be driven by the decreasing proportion of MRSA relative to MSSA; the IRR for methicillin susceptibility in $S$ aureus was 1.04 (1.03 to $1.04 \mathrm{p}<0.001)$ after adjusting for patient age, sex, organism, year, quarter, source and laboratory region.

The susceptibility of isolates to ampicillin/amoxicillin (figure 1A-C and table 2 ) declined at a rate of $2 \%$ per year for all organisms during the study period (IRR 0.98, $\mathrm{p}<0.0001$ ) and there was no evidence to suggest that this trend differed between the three organisms investigated (interaction parameter $p$ value $=0.707$ ). There was an estimated annual decrease of $10 \%$ in $H$ influenzae susceptibility to macrolides during the study period.

\section{Changes in susceptibility by age group}

Table 3 outlines susceptibility data by patient age group. Where differences in susceptibility between age groups were identified the trend was generally towards decreased susceptibility with increasing age.

Adjusted IRRs for antimicrobial susceptibility by patient age are outlined in full as a supplementary table online. There was no evidence of a difference in the susceptibility of organisms to either penicillin $(p=0.751)$ or ampicillin/amoxicillin $(p=0.500)$ after adjusting for annual and seasonal variation, patient sex, isolate source and laboratory region. However, there was some evidence that susceptibility to tetracyclines differed between age groups $(p=0.018)$ and that this difference was greatest for $S$ pneumoniae after adjusting for other relevant factors.

Co-amoxiclav susceptibility varied between age groups $(p<0.0001)$ and was lower in $S$ aureus isolated from older patients. The reported decrease in $S$ aureus susceptibility to co-amoxiclav with increasing age $(p<0.001)$ is likely to be due to the high proportion of MRSA in elderly patients as this trend is not mirrored separately for either MSSA or MRSA ( $p=0.839)$. Ciprofloxacin susceptibility varied $(\mathrm{p}<0.0001)$ and for isolates of $S$ aureus decreased from an IRR of 0.68 in patients aged $<45$ years to 0.46 in those aged $75+$ years. There was strong evidence of variation in macrolide susceptibility between age groups $(p<0.0001)$, and adjusted IRRs decreased with increasing age for all organisms, although it should be noted that $S$ aureus isolated from the youngest age group did not fit this trend.

\section{Changes in susceptibility by reporting region}

There was a small (generally $\pm 5 \%$ ) but significant $(p<0.001)$ difference in susceptibly between geographical regions for all antibiotics. However, it should be noted that the number of LRT isolates reported by each region varied markedly (range, 201 to 22810 isolates; median, 6370).

Results from the multivariable analysis suggest that the North East and London generally had lower levels of susceptibility than other regions once factors such as isolate source, patient age and sex, and pathogen had been accounted for. However, in the case of

Table 2 Adjusted incident rate ratios (IRRs) for the annual rate of susceptibility change in lower respiratory tract isolates of $H$ influenzae, $S$ aureus and $S$ pneumoniae in England, Wales and Northern Ireland (January 2007-March 2010)

\begin{tabular}{|c|c|c|c|c|}
\hline \multirow[b]{2}{*}{ Antibiotic } & \multirow[b]{2}{*}{ Organism } & \multicolumn{3}{|c|}{ Annual rate of susceptibility change } \\
\hline & & IRR † 95\% Cls & $\begin{array}{l}\text { Likelihood ratio } \\
\text { test } p \text { value }\end{array}$ & $\begin{array}{l}\text { No. of } \\
\text { observations }\end{array}$ \\
\hline $\begin{array}{l}\text { Ampicillin/amoxacillin } \\
{ }^{*} \text { Adjusted for age group and sex }\end{array}$ & All organisms & 0.98 (0.98 to 0.99$)$ & $<0.0001 \S$ & 55176 \\
\hline \multirow{3}{*}{$\begin{array}{l}\text { Tetracyclines } \\
{ }^{*} \text { Adjusted for source and sex }\end{array}$} & $H$ influenzae & $1.00(1.00$ to 1.00$)$ & & \\
\hline & $S$ aureus & $1.00(0.99$ to 1.00$)$ & $<0.0001$ & 69913 \\
\hline & $S$ pneumoniae & 0.99 (0.98 to 1.00$)$ & & \\
\hline \multirow{3}{*}{$\begin{array}{l}\text { Co-amoxiclav } \\
{ }^{*} \text { Adjusted for age group and sex }\end{array}$} & $H$ influenzae & $1.00(1.00$ to 1.00$)$ & & \\
\hline & $S$ aureus $\dagger$ & 1.09 (1.06 to 1.12$)$ & $<0.0001$ & 57895 \\
\hline & $S$ pneumoniae & $1.00(0.99$ to 1.00$)$ & & \\
\hline \multirow{3}{*}{$\begin{array}{l}\text { Ciprofloxacin } \\
{ }^{*} \text { Adjusted for age group, } \\
\text { source and sex }\end{array}$} & $H$ influenzae & $1.00(1.00$ to 1.00$)$ & $<0.0001$ & 34799 \\
\hline & $S$ aureus & $1.09(1.07$ to 1.11$)$ & & \\
\hline & $S$ pneumoniae & - & & \\
\hline \multirow{3}{*}{$\begin{array}{l}\text { Macrolides } \\
\ddagger^{*} \text { Adjusted for age group } \\
\text { and source }\end{array}$} & $H$ influenzae & $0.90(0.87$ to 0.92$)$ & & \\
\hline & $S$ aureus & $1.01(1.00$ to 1.02$)$ & $<0.0001$ & 53934 \\
\hline & S pneumoniae & $1.00(0.99$ to 1.00$)$ & & \\
\hline
\end{tabular}

*IRRs estimated using Poisson regression models with robust standard error. All models were adjusted for seasonal variation

(calendar quarter) and laboratory region as well as age group, sex and isolate source where indicated.

†Benzyl-penicillin used as a proxy for co-amoxiclav.

$\neq$ Combined susceptibility results for azithromycin, erythromycin and clarithromycin

$\S$ Likelihood ratio test $p$ value refers to the significant trend across all organisms rather than for the interaction. All the other $p$ values

outlined in the table refer to the interaction between organism and year. 
Table 3 Antimicrobial susceptibility of lower respiratory tract isolates by organism and age group, England, Wales and Northern Ireland (January 2007-March 2010)

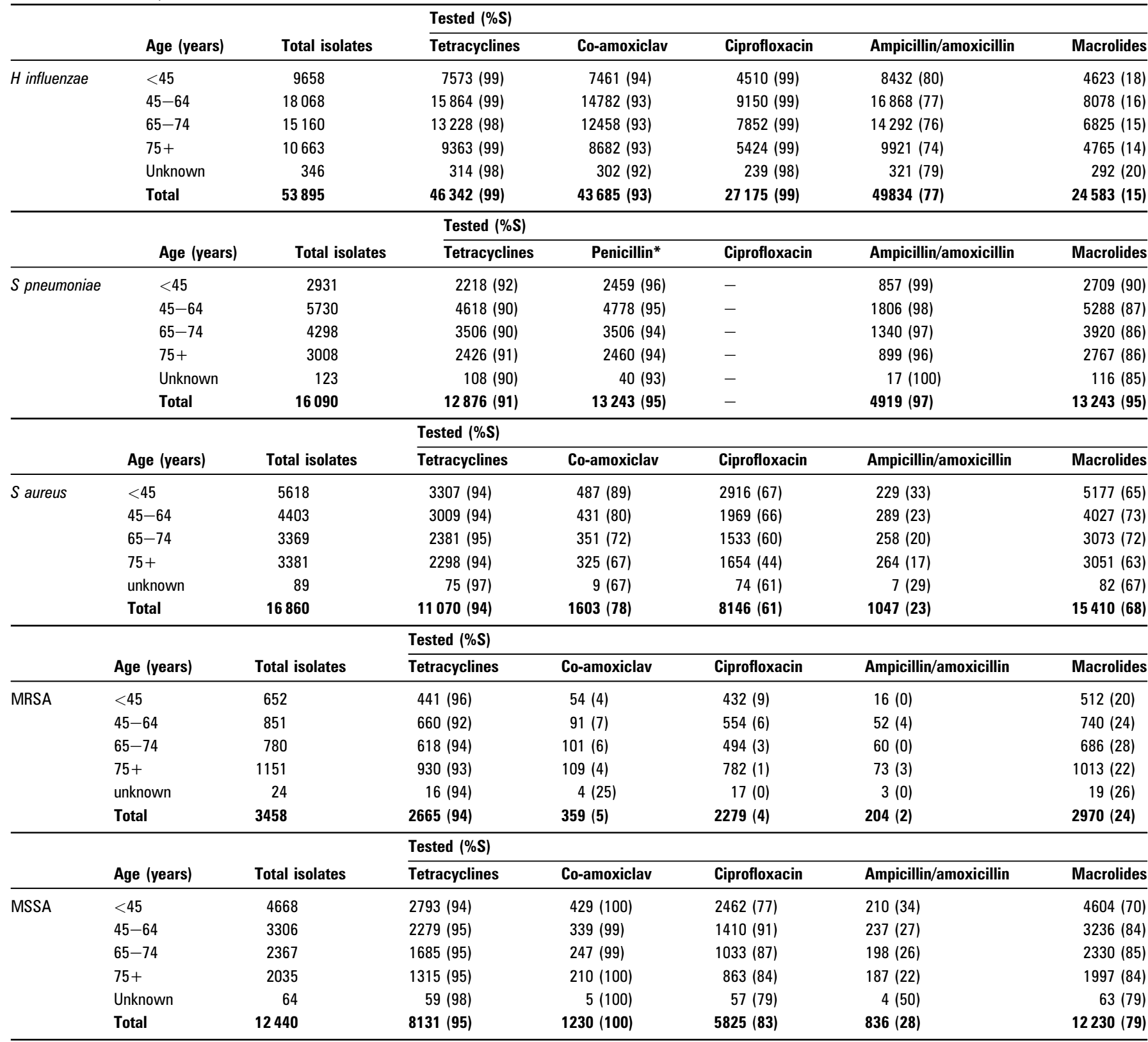

*Benzyl-penicillin used as a proxy for co-amoxiclav.

MRSA, methicillin-resistant Staphylococcus aureus; MSSA, methicillin-susceptible Staphylococcus aureus.

the North East region, lower susceptibility is likely to be a reflection of the low number of reports and potential reporting bias. The East Midlands region generally had higher levels of susceptibility in comparison with all other regions.

\section{DISCUSSION}

The 2007 guidelines identify $H$ influenzae, $S$ pneumoniae and $S$ aureus as the major bacterial pathogens for influenza-associated pneumonia. In order to test the evidence base for these guidelines, this study has focused on assessing the antimicrobial susceptibility profiles of these organisms. The data reported here support the in vitro efficacy of doxycycline or co-amoxiclav against these three pathogens. Moreover the antimicrobial susceptibility profiles of $H$ influenzae, $S$ pneumoniae and $S$ aureus obtained showed little change over time. In addition, there was little variation either geographically or between age groups for susceptibly to tetracyclines or co-amoxiclav. The validity of these data are supported by the National External Quality Assurance Scheme susceptibility testing results from UK laboratories, which show that the sensitivity of detection of resistance to tetracycline or co-amoxiclav/penicillin in these three organisms was $95-100 \%$ in 2007-2008 (personal communication V James, UK NEOAS Organiser).

LabBase is a good source of microbiological data, with comparison of MRSA bloodstream data reported to this voluntary system and to the mandatory reporting scheme indicating that up to $70 \%$ of infections in England may be captured. ${ }^{10}$ However, clinical data are not captured and, as the data reported represent a sample of all LRT isolates sent to microbiology from primary care and outpatients, it is therefore not possible to determine whether the isolates reported in this study were from 
patients with ILI-associated pneumonia. The mean GP patient age of 61 years was similar to the 66 year mean age reported for diagnosis of chronic obstructive pulmonary disease (COPD) by UK GPs between 1990 and 1997. ${ }^{11}$ The GP patient specimens included could therefore reflect the organisms associated with LRT infection in chronic lung disease. Nonetheless, these data clearly indicate that LRT isolates of the pathogens studied retain high levels of susceptibility to the antibiotics recommended in the UK guidelines.

Much GP prescribing for LRT infection is empirical, and isolates referred for laboratory testing are likely to be from patients where treatment failure has occurred or who have received prior courses of antibiotics for chronic respiratory conditions, and are therefore enriched for organisms resistant to treatment. $^{12} 13$ Thus the $92 \%$ and $96 \%$ of primary care LRT isolates found to be susceptible to co-amoxiclav or tetracycline (respectively) may well be a conservative estimate, with real susceptibility rates in the ILI-associated pneumonia population being higher. The proportion of MRSA LRT isolates reported via the scheme may also be overestimated in comparison with $H$ influenzae or $S$ pneumoniae because this organism is more frequently associated with more severe disease presentation, ${ }^{14}$ thereby increasing the likelihood that an LRT specimen will be taken.

Susceptibility to ampicillin/amoxicillin and macrolides was well below that recorded for tetracyclines or co-amoxiclav, suggesting that the former drugs are less appropriate for empirical treatment. However, susceptibility to ciprofloxacin for $H$ influenzae or ampicillin/amoxicillin for $S$ pneumoniae was high (>95\%) and these antibiotics may be preferable to tetracyclines or co-amoxiclav where the organism is known. The observed decrease in susceptibility of $H$ influenzae to ampicillin/amoxicillin from $\sim 80 \%$ at the start of the study period to $76 \%$ in the first 3 months of 2010 is supported by work from Jansen et al, who noted a similar decrease between 1997/8 and 2004/5 in a range of countries across Europe. ${ }^{15}$ The British Society for Antimicrobial Chemotherapy (BSAC) also report a similar decline in $H$ influenzae susceptibility to either ampicillin or amoxicillin (84.6\% and $77.2 \%$ susceptible, respectively, for the whole time period) between October 1999 and April 2007 in community-acquired respiratory infections in the UK and Ireland. ${ }^{16}$ During the study period (January 2007-March 2010) there were no changes in susceptibility that should cause clinicians to alter their prescribing behaviour away from the 2007 guidelines and 2009 update. The observed decrease in ampicillin/ amoxicillin susceptibility and increased $S$ aureus susceptibility to ciprofloxacin are for antibiotics already known to have suboptimal activity against the organisms associated with secondary bacterial infection following ILI.

A weakness of the current study is that other organisms may also cause ILI-associated pneumonia. ${ }^{3}{ }^{17-19}$ Streptococcus pyogenes is a particularly important respiratory pathogen which is not covered by the 2007 guidelines. ${ }^{20}$ The surveillance programme described here is being expanded to include $S$ pyogenes. Current rates of antimicrobial resistance in invasive $S$ pyogenes infection are $11 \%$ for tetracycline, while penicillin (a proxy for co-amoxiclav) resistance remains very rare $^{21}$ and suggests that these antibiotics are likely to be appropriate for LRT infections presenting in primary care, although further work is required to confirm this recommendation. It should also be noted that the number of laboratories in Wales and Northern Ireland that reported isolates during the study period is very low and the data presented in this study may therefore better reflect susceptibility trends in England. The HPA is working to improve

\section{What is already known on this topic}

Secondary bacterial pneumonia may be a complication of influenza. Published guidelines for the clinical management of influenza-like illness (ILI) in the UK recommend doxycycline or co-amoxiclav for the empirical treatment of ILI-associated pneumonia for patients in primary care.

\section{What this study adds}

This study discusses current susceptibility data for lower respiratory tract isolates of Streptococcus pneumoniae, Staphylococcus aureus and Haemophilus influenzae from hospital outpatients and GP patients in England, Wales and Northern Ireland. The results support the use of a tetracycline (doxycycline) or co-amoxiclav for the empirical treatment of secondary pneumonia in a primary care setting. There was little variation of clinical significance in antimicrobial susceptibility to tetracycline or co-amoxiclav over time, between patient age groups or geographically.

the ascertainment of LRT isolates from regions with inadequate reporting coverage. These changes should help better capture the true scale of LRT infections across regions in England, Wales and Northern Ireland.

The high in vitro susceptibility of LRT isolates of $H$ influenzae, $S$ pneumoniae and $S$ aureus to co-amoxiclav or tetracycline strongly supports the use of these antibiotics for empirical treatment of influenza-related pneumonia in a primary care setting. The susceptibility trends for each organism have remained fairly stable over time and spatially, with little variation between patient age group, thereby making any new changes easier to identify. The ongoing and improved reporting of LRT isolates will ensure that robust surveillance continues to inform national empirical treatment in the event that susceptibility patterns change.

Acknowledgements We would like to thank Neville Verlander (Statistics, Modelling \& Bioinformatics Department, Centre for Infections, Health Protection Agency) for his help and guidance with the statistical analysis presented for this study. We would also like to thank all laboratories that contributed data to the surveillance scheme, and our colleagues within the HPA Local and Regional Services and Centre for Infections. Particular thanks also go to the East of England Regional Epidemiology Unit, who played a critical role in launching the LRT surveillance scheme.

\section{Competing interests None.}

Ethical approval The HPA has National Information Governance Board for Health and Social Care approval for the collation of surveillance data in accordance with section 251 of the NHS Act 2006. No additional ethical approval was required to undertake this study.

Contributors All authors made critical contributions either to the conception of the LRT surveillance scheme or for the analysis and interpretation of the data. All authors have helped draft and revise the article and have approved the final version. The guarantor is APJ.

Provenance and peer review Not commissioned; externally peer reviewed.

\section{REFERENCES}

1. British Infection Society, British Thoracic Society, Health Protection Agency. Pandemic flu: clinical management of patients with an influenza-like illness during an influenza pandemic. Provisional guidelines from the British Infection Society, British Thoracic Society, and Health Protection Agency in collaboration with the Department of Health. Thorax 2007;62(Suppl 1):1-46. 
2. Scheiblauer H, Reinacher $\mathrm{M}$, Tashiro $\mathrm{M}$, et al. Interactions between bacteria and influenza $A$ virus in the development of influenza pneumonia. $J$ Infect Dis 1992;166:783-91.

3. Morens DM, Taubenberger JK, Fauci AS. Predominant role of bacterial pneumonia as a cause of death in pandemic influenza: implications for pandemic influenza preparedness. J Infect Dis 2008:198:962-70.

4. Benenson R, Magalski A, Cavanaugh S, et al. Effects of a pneumonia clinical pathway on time to antibiotic treatment, length of stay, and mortality. Acad Emerg Med 1999:6:1243-8.

5. Finch RG, Low DE. A critical assessment of published guidelines and other decisionsupport systems for the antibiotic treatment of community-acquired respiratory tract infections. Clin Microbiol Infect 2002;8(Suppl 2):69-91.

6. Department of Health. Pandemic H1N1 2009 influenza: clinical management guidelines for adults and children. 2009. http://www.dh.gov.uk/en/ Publicationsandstatistics/Publications/PublicationsPolicyAndGuidance/DH 107769 (accessed 4 Dec 2009).

7. Pletz MWR, McGee L, Burkhardt 0, et al. Ciprofloxacin treatment failure in a patient with resistant Streptococcus pneumoniae infection following prior ciprofloxacin therapy. Eur J Clin Microbiol Infect Dis 2005;24:58-60.

8. Fuller JD, Low DE. A review of Streptococcus pneumoniae infection treatment failures associated with fluoroquinolone resistance. Clin Infect Dis 2005;41:118-21.

9. Brown DL, Cai TT, DasGupta A. Interval estimation for a binomial proportion. Stat Sci 2001:16:101-33.

10. Pearson A, Chronias A, Murray M. Voluntary and mandatory surveillance for methicillin-resistant Staphylococcus aureus (MRSA) and methicillin-susceptible $S$ aureus (MSSA) bacteraemia in England. J Antimicrob Chemother 2009;64 (Suppl 1):i11-17.
11. Soriano JB, Maier WC, Egger $P$, et al. Recent trends in physician diagnosed COPD in women and men in the UK. Thorax 2000;55:789-94

12. Niederman MS, Mandell LA, Anzueto A, et al; American Thoracic Society. Guidelines for the management of adults with community-acquired pneumonia. Diagnosis, assessment of severity, antimicrobial therapy, and prevention. $A m \mathrm{~J}$ Respir Crit Care Med 2001:163:1730-54.

13. Woodhead M, Blasi F, Ewig S, et al. Guidelines for the management of adult lower respiratory tract infections. Eur Respir J 2005;26:1138-80.

14. Kallen AJ, Brunkard J, Moore Z et al Staphylococcus aureus community-acquired pneumonia during the 2006 to 2007 influenza season. Ann Emerg Med 2009;53:366-8.

15. Jansen W, Verel A, Beitsma $M$, et al. Longitudinal European surveillance study of antibiotic resistance of Haemophilus influenzae. J Antimicrob Chemother 2006:58:873-7.

16. Morrissey I, Maher K, Williams L, et al; on behalf of the BSAC Working Parties on Resistance Surveillance. Non-susceptibility trends among Haemophilus influenzae and Moraxella catarrhalis from community-acquired respiratory tract infections in the UK and Ireland, 1999 to 2007. J Antimicrob Chemother 2008;62(Suppl 2):ii97-103.

17. Center for Disease Control and Prevention (CDC). Bacterial co-infections in lung tissue specimens from fatal cases of 2009 pandemic Influenza A (H1N1) - United States, May-August 2009. MMWR Morb Mortal Wkly Rep 2009;58:1071-4.

18. Klugman K, Chien Y, Madhi S. Pneumococcal pneumonia and influenza: a deadly combination. Vaccine 2009;27(Suppl 3):C9-14.

19. Gill JR, Sheng ZM, Ely SF, et al. Pulmonary pathologic findings of fatal 2009 pandemic influenza A/H1N1 viral infections. Arch Pathol Lab Med 2010;134:235-43.

20. Barlow GD; BSAC Council. Swine flu and antibiotics. J Antimicrob Chemothe 2009:64:889-94.

21. HPA. Pyogenic and non-pyogenic streptococcal bacteraemias, England, Wales and Northern Ireland: 2008. Health Protection Report [serial online] 2009;3:bacteraemia.

\section{Journal club}

\section{Early palliative care in non-small cell lung cancer improves quality of life and mood, and may result in longer survival}

In this single-centre unblinded study performed in Massachusetts over 3 years, 151 outpatients with newly diagnosed metastatic non-small cell lung cancer and with a performance status of $0-2$ were randomised to either standard oncological care alone or to standard care plus early palliative care.

The study groups were broadly similar and the results were analysed on an intention-totreat basis at 12 weeks; $14 \%$ of patients in the standard care group were referred to palliative care at the discretion of the treating physician and were included in the standard care analysis. Patients in the early palliative care group had significantly higher quality of life scores as measured by various health-related quality of life scores at 12 weeks, and lower depression scores as measured by the Hospital Anxiety and Depression scale.

By the time of the final analysis, $70 \%$ of the study population had died. Of these, more of the standard care group had received invasive end of life care such as chemotherapy or emergency hospitalisation. More of the early palliative care group had resuscitation and end of life decisions documented in the notes and the early palliative care group had more days of hospice care. Despite these differences, the early palliative care group had a longer duration of survival than the standard care group although the study was not powered or designed to measure this outcome.

This study did not measure the palliative care received but, as the authors suggest, the survival difference is comparable to trials of platinum-based chemotherapy. The results are intriguing and hint towards an improvement in quality and length of life, with an associated reduction in expensive and invasive end of life treatment. At a time of rationalisation of services in the NHS and spiralling drug costs, this small study supports a role for earlier palliative care involvement.

- Temel JS, Greer JA, Muzikansky A, et al. Early palliative care for patients with metastatic non-small-cell lung cancer. $N$ Engl J Med 2010;363:733-42.

\section{B Land}

Correspondence to D B Land, SpR Medway Maritime Hospital, Gillingham, Kent, UK; davidland@nhs.uk

Published Online First 17 January 2011

Thorax 2011:66:395. doi:10.1136/thx.2010.149906 